\title{
A 10-year-old boy presented with recurrent episodes of diarrhea, low grade, irregular fever and severe wasting
}

\author{
Hazera Akter, Md. Wahiduzzaman Mazumder, A. S. M. Bazlul Karim, Subarna Rani Das and \\ Saidul Islam Sumon
}

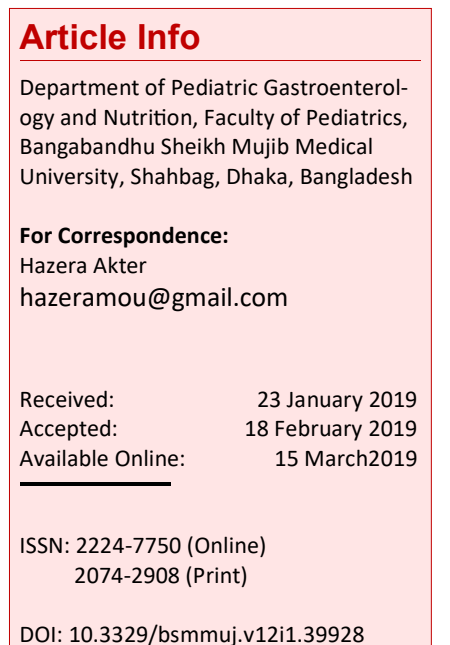

Keywords: Diarrhea; Fever; Wasting

Cite this article:

Akter $\mathrm{H}$, Mazumder MW, Karim ASMB, Das SR, Sumon SI. A 10 -year-old boy presented with recurrent episodes of diarrhea, low grade, irregular fever and severe wasting. Bangabandhu Sheikh Mujib Med Univ J. 2019; 12: 32-38.

\section{Copyright:}

The copyright of this article is retained by the author(s) [Atribution CC-By 4.0]

\section{Available at:}

www.banglajol.info

A Journal of Bangabandhu Sheikh Mujib Medical University, Dhaka, Bangladesh

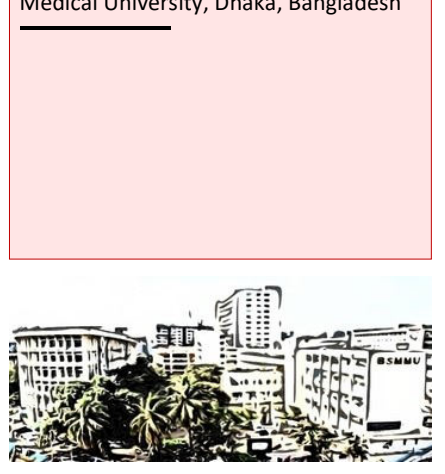

\section{Presentation of Case}

Dr. Hazera Akter: A 10-year old boy, the only issue of his deceased parents, was admitted to the department for the management of recurrent episodes of diarrhea and severe wasting for 2.5 months, low grade, irregular fever with evening rise of temperature, anorexia and repeated symptom of skin blister (Figure 1) for the last 1 year. The patient complained of chronic, painless, non-bloody, profuse watery diarrhea with a weight loss of $10 \mathrm{~kg}$ (decrease to $18 \mathrm{~kg}$ from $28 \mathrm{~kg}$ prior to illness). He also complained of cough for the last seven days. His father was a driver and died when he was 1 year of age due to an unknown disease. His mother died of tuberculosis about 4 years back. He had no history of blood or blood product transfusion, any major or minor surgery, tooth extraction and drug abuse, no history of ingestion of unboiled cows milk.

Physical examination revealed, the boy was illlooking, emaciated, febrile, having no signs of dehydration, no significant lymphadenopathy with normal vital signs, anthropometrically severely underweight, severely wasted, moderately stunted, healed lesion were present over abdomen and back, no organomegaly, no intraabdominal lump or mass, and bulk of 4 limbs were symmetrically reduced.

Other systemic examinations revealed no abnormality.

Laboratory data showed slightly reduced hemoglobin, normal leukocyte count with high

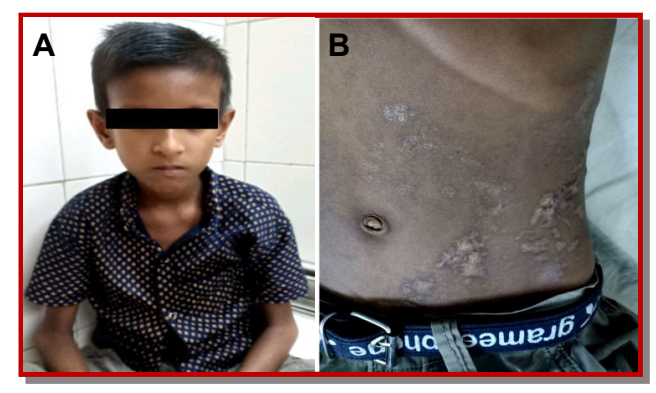

Figure 1 : Our patient (A); Healed lesion over abdomen (B) neutrophil count and lower normal lymphocyte count leading to a low absolute CD4+ count. Anti-HIV 1 and 2, plasma HIV-RNA levels were also done. His kidney functions, alanine transaminase, HBsAg, $\mathrm{tTg}-\operatorname{Ig} \mathrm{A}$, serum $\operatorname{IgA}$, IgM, IgG were normal. Urine for routine examination and culture sensitivity, stool for routine examination were also normal (Table I). His chest radiograph was normal and abdominal radiograph showed bowels were filled with gas otherwise normal. Mantoux test, sputum for acid fast bacilli and genexpert text were negative.

His ultrasonography of the whole abdomen and hepatobiliary system were also normal.

Dr. M A Taher: Barium meal showed stomach is normal in size, no ulcer crater or filling defect seen, no outlet obstruction is seen (Figure 2). No ulcer or deformity of bulb or loop was seen in duodenum. Follow through showed stomach emptied in normal time. Propulsion of meal took place through the small gut. Jejunal loops, ileal loops, ileocaecal gut were normally visualized, no organic lesion was seen.

Dr. Wahiduzzaman Mazumder: Upper GI endoscopy showed the mucosa of esophagus, fundus, body and antrum of stomach appeared normal (Figure 3). The bulb, postbulber area appeared normal. Multiple nodular swelling seen at the second part of duodenum and the histopathology report was normal.

Colonoscopy was also performed. The macroscopical findings were normal up to terminal ileum (Figure 3). The histopathological report showed non-specific colitis.

\section{Provisional Diagnosis}

Based on history, physical findings and laboratory investigations, the diagnosis was acquired immune deficiency syndrome (AIDS) - Clinical stage 3 . 
Table I

\begin{tabular}{|c|c|c|}
\hline \multicolumn{3}{|c|}{ Investigation reports of patient } \\
\hline Parameters & Findings & References \\
\hline Haemoglobin $(\mathrm{g} / \mathrm{dL})$ & 8.9 & 13.5 \\
\hline Total leukocyte count (cells/mm³) & 8,000 & $4,000-11,000$ \\
\hline \multicolumn{3}{|l|}{ Differentials } \\
\hline Neutrophil (\%) & 76 & $40-75$ \\
\hline Lymphocyte (\%) & 20 & $20-40$ \\
\hline Eosinophil (\%) & 02 & $1-6$ \\
\hline Monocyte (\%) & 02 & $2-10$ \\
\hline Serum ALT (mg/dL) & 40 & $20-60$ \\
\hline Serum creatinine $(\mathrm{mg} / \mathrm{dL})$ & 0.5 & $0.5-1.3$ \\
\hline Serum $\operatorname{Ig} A(g / L)$ & 1.79 & $0.7-4.0$ \\
\hline Serum IgM (g/L) & 15.8 & $7-16$ \\
\hline Serum IgG $(g / L)$ & 0.91 & $0.4-2.3$ \\
\hline $\operatorname{tTg}-\operatorname{IgA}(\mathrm{u} / \mathrm{mL})$ & 7.10 (Negative) & Up to 60 \\
\hline HBsAg & Negative & \\
\hline Mantoux test & Negative (2 mm) & \\
\hline Sputum for AFB & Negative & \\
\hline Gene-Xpart & Negative & \\
\hline Anti HIV 1 and 2 & Positive & \\
\hline Absolute CD4+ count (cells / mm³) & 256 & $500-1500$ \\
\hline HIV-RNA levels & 11650 copies/mL & Not detected \\
\hline Urine $\mathrm{R} / \mathrm{M} / \mathrm{E}$ and $\mathrm{C} / \mathrm{S}$ & $\begin{array}{l}\text { Pus cell, RBC, Mac- } \\
\text { rophage-Nil }\end{array}$ & \\
\hline Stool examination & $\begin{array}{l}\text { No red blood cells } \\
\text { or leukocytes }\end{array}$ & \\
\hline
\end{tabular}

\section{Differential Diagnosis}

Dr. A. S. M. Bazlul Karim:

\section{Intestinal tuberculosis}

Chronic diarrhea is one of the most common presentations of intestinal tuberculosis. Diarrhea due to mucosal ulceration may be seen in gastrointestinal tuberculosis 1,2 particularly in patients with ileocecal with or without jejunal tuberculosis. Diarrhea is observed in as high as $76 \%$ of patients with intestinal tuberculosis. Organisms involved in intestinal tuberculosis are Mycobacterium tuberculosis and M. bovis. 3 The primary intestinal tuberculosis is usually caused by $M$. bovis. It commonly involved in the ileocecal region (64-90\%) particularly the terminal ileum followed by the jejunum $(16 \%)$. The final clinical diagnosis is based on the combination of clinical history (fever, anorexia and weight loss, diarrhea, hematochezia and malabsorption, abdominal pain, and distention and vomiting) with endoscopic studies, culture and polymerase chain reaction (PCR) for M. tuberculosis, histopathological findings, radiological investigations (barium meal, follow through, enema) and response to therapy. The histological hallmarks of tuberculosis are confluent, caseating granulomas containing acid fast bacilli and surrounded by lymphoid tissue.1, 3,4 These are found in all the layers of intestinal wall and in the regional lymph node. 6 Abdominal radiographs may show signs of obstruction (presence of multiple air-fluid levels and dilated loops of the gut) and/or perforation (air under diaphragm), calcification in the abdominal lymph nodes. Contrast studies are more beneficial to demonstrate the intestinal mucosa. For an accurate diagnosis, a high clinical index of suspicion is also necessary. $\cdot$ - -7

\section{Inflammatory bowel disease}

Dr. Akter: Chronic diarrhea is a common presenting feature of inflammatory bowel disease. In the case of inflammatory bowel disease, bloody diarrhea with abdominal pain occurs. But our patient has watery diarrhea not associated with passes of blood and abdominal pain. He has no extraintestinal manifestation, any perianal lesion which is common in inflammatory bowel disease mainly Chron's disease. Inflammatory bowel disease is diagnosed by a good clinical history with colonoscopical and biopsies findings (macroscopically shows bowel
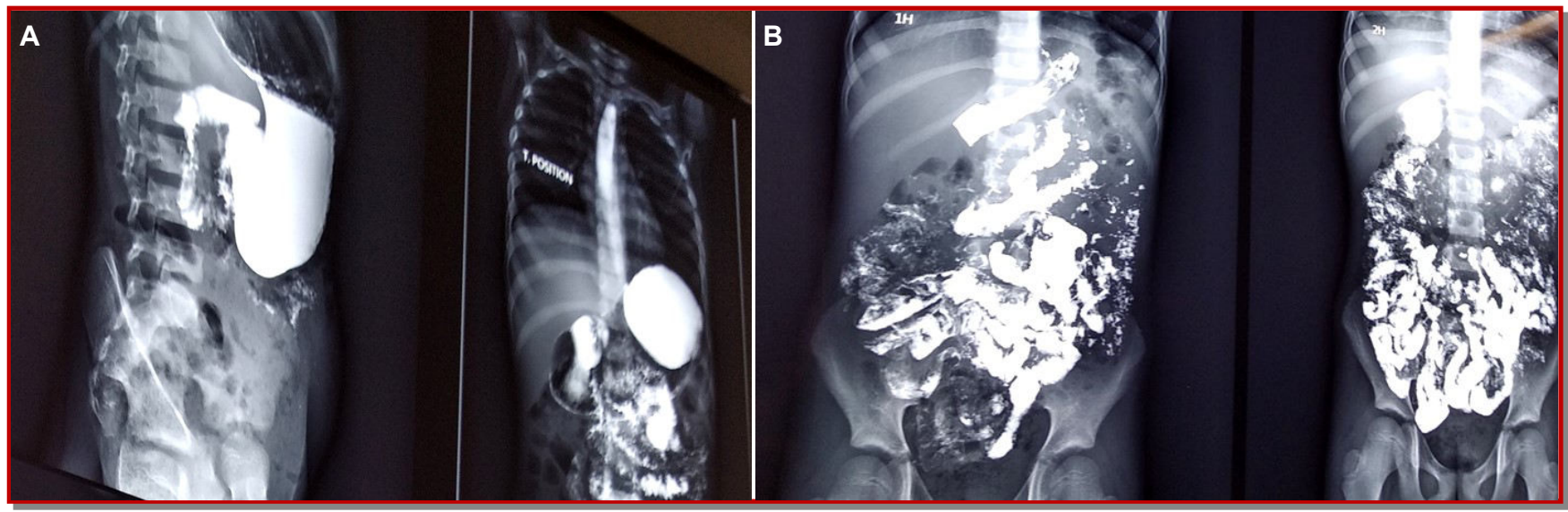

Figure 2: Barium meal showing normal findings (A); Barium follow through of small bowel (B) 

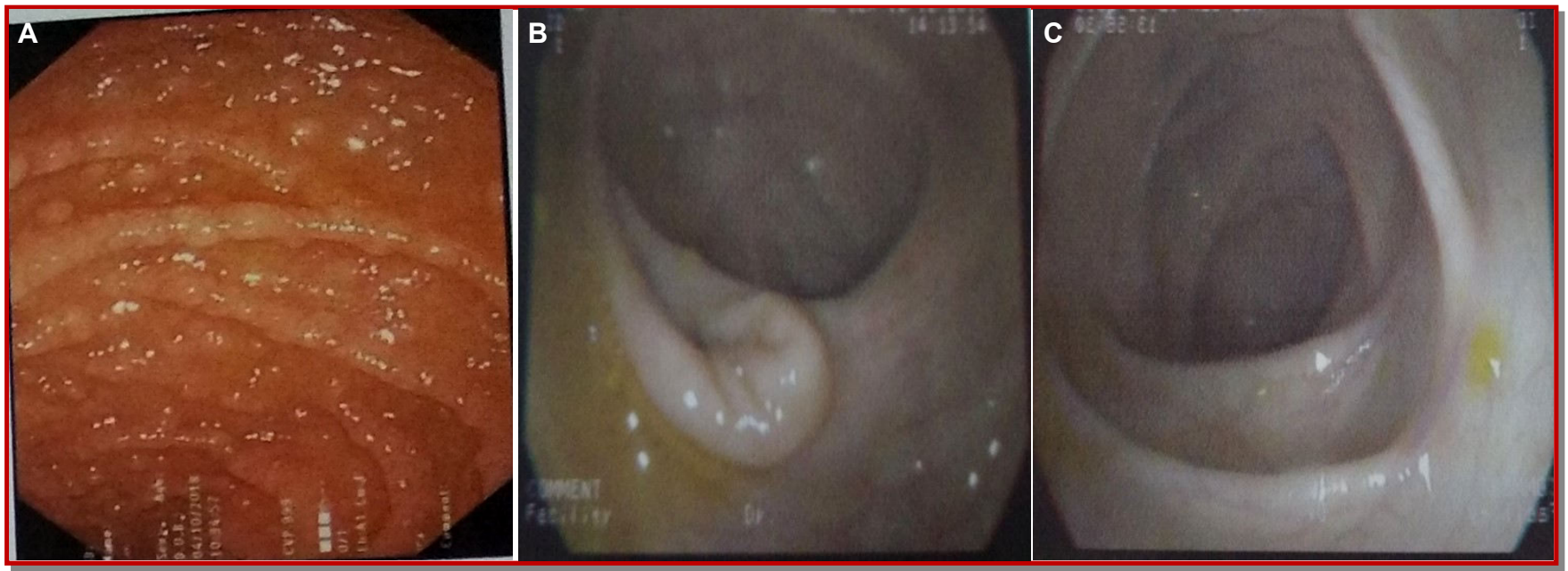

Figure 3: Multiple nodular swelling in second part of duodenum seen during endoscopy (A); Colonoscopic picture of cecum and colon showing no abnormality (B and $\mathrm{C}$ )

wall thickening, skip lesions and strictures, adhesions, fistulae, sinuses. Mucosal aphthous ulcers are seen at an early stage and deep longitudinal, fissuring ulcers are characteristic of Chron's disease. Smaller longitudinal ulcers separate edematous or uninvolved mucosa and create a cobblestone appearance. Microscopically aphthous ulcers over lymphoid follicles, fissuring ulcers that extend into the muscularis propria or deeper, distortion of the mucosal architecture, pyloric metaplasia, cryptitis and crypt abscess are common features. Another characteristic feature is non-ceaseating granuloma). Cultures and barium study (aphthous ulcers, segmental colitis and cobblestoning ) are also helpful for diagnosis.?

\section{Dr. Hazera's Diagnosis}

Acquired immune deficiency syndrome (AIDS) Clinical stage 3

\section{Discussion}

Dr. Archana Shrestha Yadav: We know that the causative agent is the human immune virus (HIV). How this virus can be transmitted?

Dr. Akter: HIV is spread only in certain body fluids from a person with HIV. These body fluids are blood, semen, pre-seminal fluids, rectal fluids, vaginal fluids and breast milk. HIV transmission is only possible if these fluids come in contact with a mucous membrane or damaged tissue or are directly injected into the bloodstream (from a needle or syringe). .8

Dr. Kamrun Nahar: What are the risk factors of HIV infection?

Dr. Subarna Rani Das: Having unprotected vaginal or anal sex with someone who is HIV positive. a) Injecting drugs and sharing needles, syringes, or other drug equipment with someone who is HIV positive or whose HIV status is unknown; b) Exchanging sex; c) People suffering from hepatitis, tuberculosis, sexually transmitted disease (STD) such as syphilis.

Dr. Md. Benzamin: Can I get HIV from mosquito bites or scratched by an HIV infected person?

Dr. Saidul Islam Sumon: No. HIV is not spread by mosquitoes, ticks, or any other insects. It is not spread through saliva and there is no risk of transmission from scratching because no body fluids are transferred between people when scratched by someone. You cannot get HIV from casual contact e.g., Hugging, closed-mouth or social kissing, shaking hands, sharing toilets, sharing dishes or the sneezing and coughing of an infected person.

Dr. Luthfun Nahar: How well does the HIV survive outside the body?

Dr. Akter: HIV does not survive long outside the human body and it cannot reproduce without a human host. 8

Dr. Sayma Munmun: Is there any connection between HIV and other sexually transmitted diseases?

Dr. Akter: Yes, there is a connection. One person having another STD (gonorrhea, chlamydia, syphilis, Trichomoniasis, human papillomavirus (HPV) genital herpes, and hepatitis) has three times more chance to get or transmit HIV to others.

Dr. Benzamin: How can we prevent the transmission of HIV from mother to baby?

Dr. Sumon: All pregnant women with HIV should start taking HIV medicines as soon as possible to prevent mother to baby transmission of HIV. 
Women who are already taking HIV medicines should continue taking those HIV medicines throughout their pregnancies. The risk of mother-to -baby transmission of HIV is more during the vaginal delivery when a baby passes through the birth canal and is exposed to any HIV in the mother's blood and other fluids. A scheduled cesarean delivery can reduce the chance of motherto baby transmission of HIV in a HIV infected women. Cesarean delivery is scheduled at the $38^{\text {th }}$ week of pregnancy or 2 weeks before a woman's expected date. By taking these measures the risk of mother to child transmission can be lowered $2 \%$ or less. $.8,9$

Dr. Subir Ananda Biswas: Can I get vaccinated to prevent HIV?

Dr. Akter: No therapeutic or preventive HIV vaccines have been approved by the Food and Drug Administration (FDA) but research is ongoing.

Dr. Rana Kumar Biswas: When to start antiretroviral therapy in children?

Dr. Subarna Rani Das: Antiretroviral therapy should be initiated in all children living with HIV, regardless of $\mathrm{WHO}$ clinical stage or at any CD4 cell count. a) Children $<1$ year; b) Children younger than 5 years of age with who clinical stage 3 or 4 or CD4 count $<750$ cells $/ \mathrm{mm}^{3}$ or CD4 percentage $<25 \%$; c) Children older than 5 years of age with who clinical stage 3 or 4 or CD4 count $<350$ cells/ $\mathrm{mm}^{3} \cdot \underline{9,10}$

Dr. Mohuya Mondal: What are the criteria for the diagnosis of HIV infection in a pediatric patient?

Dr. Saidul Islam Sumon: WHO case definition for HIV infection in pediatric patients.

Children $\geq 18$ months: Positive HIV antibody testing (rapid or enzyme immunoassay) and confirmed by a second HIV antibody test and/or positive virological test for HIV or its components (HIVRNA or HIV-DNA or ultrasensitive HIV p24 antigen) confirmed by a second virological test obtained from a separate determination.

Children $<18$ months: Positive virological test for HIV or its components (HIV-RNA or HIV-DNA or ultrasensitive HIV p24 antigen) confirmed by a second virological test obtained from a separate determination taken more than four weeks after birth. Positive HIV antibody testing is not recommended for definitive or confirmatory diagnosis of HIV infection in children until 18 months of age. $\underline{11}$

Dr. Naznin Sarker: What management is given to your patient?

Dr. Akter: At first we counsel the parents about the nature and course of the disease, available treatment and prognosis. Then we managed the patient with a balanced diet and supportive care with oral rehydration solution, vitamins and minerals. We advised the patient to maintain personal hygiene and avoid to eat raw undercooked food, unsafe water, playing with pet/farm animal. After confirmation of diagnosis, we start tablet tenofovir, tablet lamivudine, tablet efavirenz, and syrup co-trimoxazole.

Dr. Fathema: What are the gastrointestinal malignancies commonly associated with AIDS?

Dr. Akter: HIV-associated gastrointestinal malignancies are Kaposi sarcoma, AIDS-related anal squamous cell carcinoma/anal intraepithelial neoplasia (SCC/AIN) and non-Hodgkin lymphoma (AIDS-NHL), Burkitt's and Burkitt-like lymphomas and diffuse large- $\beta$-cell lymphomas (DLBL) are the subtypes that most likely affect the gastrointestinal tract in HIV-infected patients. $\underline{12,13}$

Dr. Wahiduzzaman Mazumder: Chronic diarrhea is one of the most common gastrointestinal symptom of AIDS, classic enteric pathogens and different opportunistic agents both are responsible for this event. 14,15 Chronic, well-established diarrhea, persisting longer than 1 month, for which no infective agent can be identified after a complete evaluation, in patient with documented advanced HIV infection is termed as AIDS enteropathy. $\underline{.16-19}$ Here, malabsorption and severe wasting occur together that's why it is also called wasting disease. $\underline{20}$ Chronic diarrhea affecting nearly $60-80 \%$ of patients with the HIV. It is a more common manifestation close to $100 \%$ of advanced HIV disease in developing countries. $\underline{.21,22}$

AIDS enteropathy was first described by Kotler and colleagues in 1984 in patients with no identifiable pathogen but with diarrhea and malnutrition. The mucosal surface of gastrointestinal tract acts as an important barrier that protects against pathogenic organisms. But in HIV infected individual normal defense mechanisms are destroyed (manifest as villous atrophy, crypt hyperplasia, and villous blunting), leading to a wide range of clinical and pathogenic consequences. The pathophysiology of the AIDS enteropathy is a complex, $\underline{21,23}$ the exact mechanism is unknown. The immunologic alteration can affect the gut in different ways. First one is the disequilibrium of the total immune system in AIDS patients, resulting from the depletion of CD4 helper $\mathrm{T}$ lymphocytes and the diminution in the ratio of $\mathrm{CD} 4$ to $\mathrm{CD} 8$. As a consequence of these events, frequent opportunistic infections occur in the gut with enteric organisms. $\underline{14}$ In healthy individuals, enteric secretory IgA prevents the adherence of potential pathogens to the gut epithelial surface but in HIV-infected patients, this event does not occur. So, cellular immune responses are impaired in the gut-associated lymphoid tissue. $.22,24$

Another explanation is the HIV itself that does not seem to directly infect enterocytes. It may have an 
indirect effect via cytokines and multiple inflammatory mediators, released from the infected mononuclear cells, enterochromaffin cells and lymphocytes of the lamina propria. The intestinal mucosa of HIVinfected individuals is rich in cytokines like TNFa, IL-1 $\beta$, and IFNY. These are responsible for the induction of active $\mathrm{Cl}^{-}$secretion or could directly affect the integrity of the mucosal surface, leading to villous atrophy and crypt atrophy or hypertrophy and possibly resulting in malabsorption, diarrhea, and weight loss. $\underline{14}$

Some studies show that the incidence of epithelial hypoproliferation and dysmaturation has been found to be higher in HIV-infected patient without secondary intestinal infection. $\underline{25}$

The clinical presentations mainly depend on which part of the gastrointestinal tract involved. Candida and Herpis commonly involved the oral cavity, esophagus and stomach, cause loss of appetite, dysphagia and weight loss. When organisms involved the intestine, it causes diarrhea. Small bowel diarrhea is characterized by large volume postprandial diarrhea (more than one per day) with associated dehydration, paraumbilical pain and weight loss. There is absence of tenesmus (painful defecation), white cells or gross blood in the stools and improved with fasting. Large bowel diarrhea is characterized by frequent, small-volume stools without dehydration, accompanied by lower quadrant or suprapubic abdominal pain and visible blood and mucus may be present in the stool.21,26

The most common opportunistic pathogens identified among the HIV patient with chronic diarrhea are the microsporidia, cytomegalovirus, cryptosporidia and M. avium complex.

Microsporidiosis may cause profuse watery diarrhea, weight loss, and abdominal pain, but no fever or loss of appetite. It is responsible for 15 to $20 \%, 21$ 14 to $60 \% \underline{27}$ of all chronic unexplained diarrhea in patients with AIDS, especially those with CD4 <100 cells $/ \mathrm{mm}^{3}$. Diagnosis of microsporidiosis can be made by the examination of unconcentrated stool specimens with Weber's modified trichrome stain or small bowel biopsy with hematoxylin and eosin stain or transmission electron microscopy. Transmission electron microscopy has been considered the gold standard. Several recent studies have shown the sensitivity of the hematoxylin and eosin stain to be $83 \%$ with a specificity of $95 \%$ as compared to transmission electron microscopy. No specific treatment is available in patient with microsporidiosis, treated with the combination of highly active antiretroviral therapy and albendazole $400 \mathrm{mg}$ orally twice daily and continue until CD4 Tcell count $\geq 200$ cells/uL for more than 6 months after initiation of highly active antiretroviral therapy along with empiric antidiarrheal agents and nutritional support. $\underline{21,26,27}$
Cryptosporidium parvum is a protozoan parasite which causes chronic, intractable diarrhea in AIDS patient; especially those with a CD4 count $<150$ / $\mathrm{mm}^{3}$ whereas HIV-infected patient with CD4 counts $>200$ cells $/ \mathrm{mm}^{3}$ it causes self-limited diarrheal illness. Cryptosporidial infection has a predilection for the proximal small bowel and causes profuse, watery, non-bloody diarrhea leading to fluid and electrolyte depletion. Diarrhea is usually accompanied with paraumbilical abdominal pain, nausea, and vomiting. It also causes non-specific thickening of the duodenum, jejunum and proximal ileum. The diagnosis of cryptosporidiosis is usually made with acid fast stain of concentrated stool and a specific enzyme immunoassay or immunofluorescence antibody test for cryptosporidia. By CT scan, we can identify multiple loops of fluid-filled and thickened small bowel loop. Nitazoxanide along with antiretroviral treatment may be used for AIDS patient with cryptosporidiosis recommended by Centers for Disease Control and Prevention. Combination of azithromycin plus paromomycin can also be used. Other drugs including spiramycin, azithromycin, clarithromycin, roxithromycin, letrazuril, and bovine immune concentrate are under research. $21,26,27$

Cytomegalovirus is a frequent cause of diarrhea in AIDS patients. It may be responsible for 10 to $20 \%$ of debilitating chronic diarrheal illness and also causes colitis in advanced HIV infection where CD4 count is almost always $<50$ cells $/ \mathrm{mm}^{3}$. Patient with cytomegalovirus infection present with chronic watery diarrhea in $80 \%$, abdominal pain in $50 \%$ and fever in $39-80 \%$. Esophageal ulcerations may be the only manifestation. It may be associated with bile duct obstruction and intrahepatic sclerosingcholangiitis (AIDS cholangiopathy). The diagnosis is made by the demonstration of intranuclear cytomegalovirus inclusions (Cowdry Type A) on colonic biopsy; multiple biopsies may be required. Cytomegalovirus treated with intravenous ganciclovir or foscarnet oral valganciclovir used as maintenance therapy. $.11,26,27$

M. avium complex infection is associated with diarrhea, weight loss, fever, severe anemia and generalized abdominal pain, particularly right upper quadrant pain related to hepatic infiltration. The diagnosis of M. avium complex is made by acid fast bacilli culture of blood or stool, while endoscopic biopsy demonstrate foamy macrophages in the lamina propria, containing numerous acid fast bacilli. Combination chemotherapy (particularly ethambutol and clarithromycin) are used for the treatment of $M$. avium complex in the gut. $\underline{\text { 21, 26-28 }}$

For the diagnosis of chronic diarrhea after taking a detailed history and thorough physical examination, we will do some investigations. At first, we send multiple stool samples (at least 3) for the culture for Shigella, Salmonella, Campylobacter, ova and parasite examination, assay for Clostridium 
difficile toxin, smears for acid fast bacilli and culture for $M$. avium complex. Routine blood culture and $M$. avium complex blood culture should be done if the patient is febrile. If no pathogen is identified by the above mentioned investigations, endoscopy is indicated, especially the patient with fever, weight loss, and CD4 count $<200$ cells $/ \mathrm{mm}^{3}$. If there is the presence of distal colorectal symptom or fecal leukocytes, colonoscopy is indicated. $\underline{29}$

In the treatment of HIV-related diarrhea along with highly active antiretroviral therapy, we should include general measures such as maintaining adequate hydration and good nutrition. Patients should be encouraged to take adequate sugar and electrolyte-rich fluids. If necessary, an elemental diet or nutrient formula containing medium chain triglycerides can be used but the patient must be cautioned against the use of caffeine, fat and lactose containing food; also increases the soluble fiber consumption. The benefit of the use of antidiarrheal agent like loperamide, diphenoxylate with atropine, codeine phosphate are controversial. Multiple nonstandard therapies including non-steroidal antiinflammatory agents, parenteral hyperalimentation, and the use of somatostatin analogs such as octreotide are also recommended. If any enteric pathogen is identified, then treatment should be done accordingly.

\section{Follow-up}

The patient was followed up every 6 months. Clinically the improvement of presenting complaints like diarrhea, fever, weight loss were observed. Some of the laboratory tests like CD4 count, complete blood count, renal function tests, liver function tests were done. Along with these we do the HIV RNA level at 4 weeks and 3-4 months after initiation of therapy, and then 3-6 monthly.

\section{Final Diagnosis}

Acquired immune deficiency syndrome (AIDS)Clinical stage 3

\section{References}

1. Sood R. Diagnosis of abdominal tuberculosis: Role of imaging. J Indian Acad Clin Med. 2001; 2: 169-77.

2. Pulimood AB, Amarapurkar DN, Ghoshal U, Phillip M, Pai CG, Reddy DN, Nagi B, Ramakrishna BS. Differentiation of Crohn's disease from intestinal tuberculosis in India in 2010. World J Gastroenterol. 2011; 17: 433-43.

3. Concepcion NDP, De Lima GU. Chronic diarrhea: An unusual symptom of gastrointestinal tuberculosis. WPFI TB Corner. 2016; 2: 1-10.
4. Mandavdhare HS, Singh H, Sharma V. Recent advances in the diagnosis and management of abdominal tuberculosis. EMJ Gastroenterol. 2017; 6: 52-60.

5. Sarkar DN, Amin R, Mohammed H, Azhar MA, Faiz MA. Abdominal tuberculosis: A review. Bangladesh J Med. 2011; 22: 51-59.

6. Ali A, Ans M, Munir MK, Rehman S, Asghar N. Diagnostic approaches and treatment of abdominal tuberculosis in tertiary care settings. P J M H S. 2018; $12: 630-33$

7. Chugh SN, Jain V. Abdominal tuberculosis: Current concepts in diagnosis and management. 2007; 600-08. 10.5005/jp/books/12086-102.

8. HIV/AIDS, Centers for disease control and prevention. https://www.cdc.gov/hiv/basics/ index.html.

9. World Health Organization. Guide line on when to start antiretroviral therapy and on pre-exposure prophylaxis for HIV. 2015. Geneva: (http:// www.who.int/hiv/pub/guidelines/earlyrelease$\operatorname{arv} /$ en).

10. World Health Organization. Consolidated guidelines on the use of antiretroviral drugs for treating and preventing HIV infection: Recommendations for a public health approach Geneva. 2013. (http://www.who.int/hiv/pub/ guidelines/arv2013/download/en

11. World Health Organization. WHO case definitions of HIV for surveillance and revised clinical staging and immunological classification of HIV-related disease in adults and children. 2007.

12. Jensen BE, Oette M, Haes J, Häussinger D. HIVAssociated gastrointestinal cancer. Oncol Res Treat. 2017; 40: 115-18.

13. Ho-yen C, Chang F, Van der Walt J, Lucas S. Gastrointestinal malignancies in HIV-infected or immunosuppressed patients: Pathologic features and review of the literature. Adv Anat Pathol. 2007; 14: 431-43.

14. Brandi G, Calabrese C, Manfredi R, Pisi AM, Febo GD, Hakim R, Cenacchi G, Biasco G. HIV Enteropathy: Undescribed ultrastructural changes of duodenal mucosa and their regression after triple antiviral therapy: A case report. Dig Dis Sci. 2005; 50: pp 617-22.

15. Singla R, Hari S, Sharma SK. Diffuse small bowel thickening in AIDS patient: A case report. BMC Infect Dis. 2010; 10: 310 .

16. Greenson JK, Belitsos PC, Yardley JH, Bartlett JG. AIDS enteropathy: Occult enteric infections and duodenal mucosal alterations in chronic diarrhea. Ann Intern Med. 1991; 114: 366-72.

17. Simon D, Kotler DP, Brandt LJ. Chronic unexplained diarrhea in human immunodeficiency virus infection: Determination of the best diagnostic approach. Gastroenterology 1996; 111: 269-70. 
18. Beaugerie L. HIV enteropathy. Ann Gastroenterol Hepatol. 1993; 29: 19-23.

19. Lew EA, Poles MA, Dieterich DT. Diarree associate a infezione da HIV. Gastroenterol Clin. 1997; 26 : 295-317.

20. Bjarnason I, Sharpstone DR, Francis N, Marker A, Taylor C, Barrett M, Macpherson A, Baldwin C, Menzies IS, Crane RC, Smith T, Pozniak A, Gazzard BG. Intestinal inflammation, ileal structure and function in HIV. AIDS. 1996; 10: 1385-91.

21. Zacharof AK. AIDS-related diarrhea, pathogenesis, evaluation and treatment. Ann Gastroenterol. 2001; 14: 22-26.

22. Elfstrand L, Florén $\mathrm{CH}$. Management of chronic diarrhea in HIV-infected patients: Current treatment options, challenges and future directions. HIV/AIDS (Auckl). 2010; 2: 219-24.

23. Assimakopoulos SF, Dimitropoulou D, Marangos M, Gogos CA. Intestinal barrier dysfunction in HIV infection: Pathophysiology, clinical implications and potential therapies. Infection 2014; 42: 951-59.

24. Mathewson JJ, Jiang ZD, DuPont HL, Chintu C, Luo N, Zumla A. Intestinal secretory IgA immune response against human immunodeficiency virus among infected patients with acute and chronic diarrhea. J Infect Dis. 1994; 169: 614-17.

25. Lutz NW, Yahi N, Fantini J, Cozzone PJ. Perturbations of glucose metabolism associated with HIV infection in human intestinal epithelial cells: A multinuclear magnetic resonance spectroscopy study. AIDS. 1997; 11: 147-55.

26. Cello JP, Lukejohn W. Idiopathic AIDS Enteropathy and treatment of gastrointestinal opportunistic pathogens. Gastroenterology 2009; 136: 1952-65.
27. Oldfield EC $3^{\text {rd }}$. Evaluation of chronic diarrhea in patients with human immunodeficiency virus infection. Rev Gastroenterol Dis. 2002; 2: 176-88.

28. Ahsan SMA, Ahsan T, Kabir H, Jinia TJ. Patient with a short history of lower abdominal pain, anorexia and fever. Bangabandhu Sheikh Mujib Med Univ J. 2018; 11: 82-88.

29. Wilcox CM, Schwartz DA, Cotsonis G, Thompson SE 3rd. Chronic unexplained diarrhea in human immunodeficiency virus infection: Determination of the best diagnostic approach. Gastroenterology 1996; 110: 30-37.

30. Brenchley JM, Douek DC. HIV infection and the gastrointestinal immune system. Mucosal Immunol. 2008; 1: 23-30.

31. Clay PG, Crutchley RD. Noninfectious diarrhea in HIV seropositive individuals: A review of prevalence rates, etiology and management in the era of combination antiretroviral therapy. Infect Dis Ther. 2014; 3: 103-22.

32. Dikman AE, Schonfeld E, Srisarajivakul NC, Poles MA. Human immunodeficiency virus-associated diarrhea: Still an issue in the era of antiretroviral therapy. Dig Dis Sci. 2015; 60: 2236-45.

33. Johanson JF, Sonnenberg A. Efficient management of diarrhea in the acquired immunodeficiency syndrome (AIDS): A medical decision analysis. Ann Intern Med. 1990; 112: 942-48.

34. Cello JP, Grendell JH, Basuk P, Simon D, Weiss L, Wittner M, Rood RP, Wilcox CM, Forsmark CE Read AE, Satow JA, Weikel CS, Beaumont C. Effect of octreotide on refractory AIDS-associated diarrhea: A prospective, multicenter clinical trial. Ann Intern Med. 1991; 115: 705-10. 